\title{
Two-Dimensional Configurations of Unidimensional Stimulus Sets in Nonmetric Multidimensional Scaling
}

\author{
Mark L. Davison and Marsha Hearn \\ University of Minnesota
}

\begin{abstract}
When unidimensional stimulus sets are subjected to a nonmetric scaling in two dimensions, the stimuli frequently form a $\mathrm{C}$ - or $\mathrm{S}$-shaped configuration. In simulated unidimensional data scaled in two dimensions, stimuli formed a $\mathrm{C}$-shaped configuration when the monotone function relating distances to dissimilarity data was negatively accelerating. They formed an Sshaped configuration when the monotone function was positively accelerating. Results suggest that when unidimensional stimulus sets are scaled in two dimensions using a rational starting configuration, the nature of the two-dimensional configuration can indicate the general form of the function mapping psychological dissimilarity, represented as distance in the scaling model, onto the observed response scale. Index terms: data transformations, multidimensional scaling, paired comparisons, proximity data, unidimensional scaling, unidimensionality.
\end{abstract}

Several authors have noted that when unidimensional stimulus sets are scaled in two dimensions, the stimuli frequently form a C- or S-shaped configuration (Davison, 1983; Heiser, 1981; Kruskal \& Wish, 1978; Shepard, 1974). Some authors, such as Heiser (1981) and Kruskal and Wish (1978), referred to the $\mathrm{C}$-shaped configuration as the "horseshoe" configuration. In this study, the shape of the two-dimensional configuration was examined to determine whether it indicates the general

APPLIED PSYCHOLOGICAL MEASUREMENT

Vol. 13, No. 4, December 1989, pp. 329-334

(C) Copyright 1989 Applied Psychological Measurement Inc.

0146-6216/89/040329-06\$1.55 form of the monotone function mapping distances in the scaling model onto the observed data.

This question grew out of a conjecture as to why unidimensional stimulus sets sometimes form a $\mathrm{C}$ shaped configuration and at other times form an $\mathbb{S}$ shaped configuration. The hypothesis was that if the monotone function relating distances to data were negatively accelerating, the unidimensional stimulus set would form a $\mathrm{C}$ in two dimensions. Alternatively, if the monotone function were positively accelerating, the hypothesis was that the two-dimensional configuration would form an $\mathbf{S}$ shaped curve. It is assumed that the data are scored so as to be dissimilarities; that is, the monotone function mapping distances onto the data is assumed to be monotone increasing.

To understand the reasoning benind the conjecture, consider Figure 1. If the monotone function is negatively accelerating (the dotted line in Figure 1), the large distances will be smaller (relative to small distances) than they would be if the monotone function were linear (the solid line in Figure 1). Figure 2 a shows how points along a line can be folded to make the large distances relatively smaller. (The larger distances correspond to those between points at opposite ends of the line.) By bending the opposite ends of the line toward each other, the larger distances can be made relatively smaller. Hence distances along a C-shaped curve can closely approximate dissimilarity data for a unidimensional stimulus sef when the monotone transformation re- 


\section{Figure 1}

Linear (Solid Line), Positively Accelerating (Dashed Curve), and Negatively Accelerating (Dotted Curve) Monotone Increasing Functions

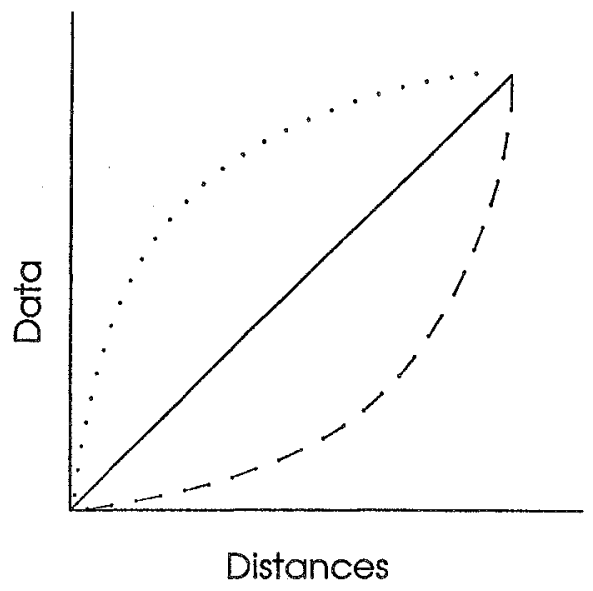

lating distances to the dissimilarity data is negatively accelerating.

If the monotone function is positively accelerating (the dashed line in Figure 1), the large distances will be larger (relative to small distances) than they would be if the monotone function were linear. Figure $2 \mathrm{~b}$ shows how points along a line can be folded to make the large distances relatively larger. By bending points at opposite ends of the line away from each other, the large distances can be expanded relative to the small distances. Hence distances along an S-shaped curve can closely approximate dissimilarity data when the monotone function is positively accelerating.

In short, it was hypothesized that when unidimensional stimulus sets are scaled in two dimensions, the shape of the configuration is related to the form of the monotone function mapping distances onto the dissimilarity data. Given dissimilarity data, a negatively accelerating function gives rise to a C-shape. A positively accelerating function gives rise to an $\mathrm{S}$-shape.

\section{Method}

Distances between 30 evenly spaced points along
Figure 2

(a) End Points of a Line Folded Toward Each Other So As to Approximate a Negatively Accelerating Monotone Function

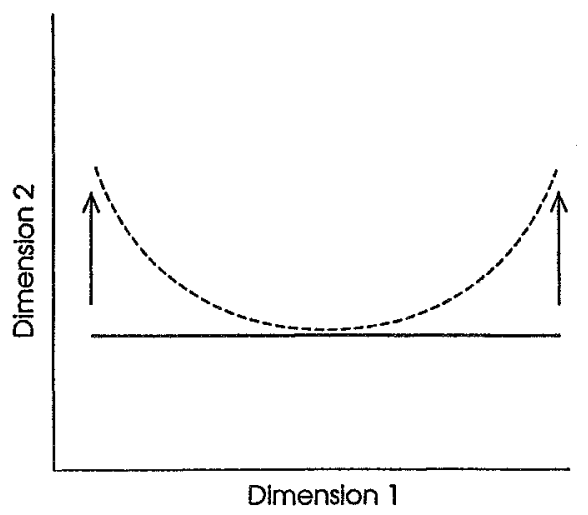

(b) End Points of a Line Folded in Opposite Directions So As to Approximate a Positively Accelerating Monotone Transformation

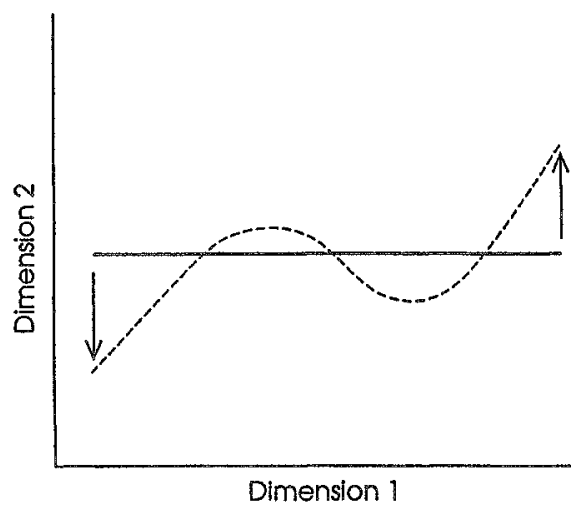

a line were generated. Seven dissimilarity datasets were created by raising the distances to powers of $.25, .33, .50,1.00,2.00,3.00$, and 4.00 . The first three powers correspond to negatively accelerating monotone functions and the last three to positively accelerating monotone functions. The 1.00 power corresponds to a linear function for which the acceleration is 0.0 . The resulting seven datasets were scaled in one and two dimensions using KYST (Kruskal, Young, \& Seery, 1973), KYST's TORSCA starting configuration, and default options. 


\section{Results}

For the negatively accelerating functions--powers of $.25, .33$, and .50 -all of the configurations were C-shaped or horseshoe-shaped. Because the configurations were so similar, only one of them is shown in Figure 3: the configuration for distances raised to the power 33 . For positively accelerating functions-powers of $2.00,3.00$, and 4.00 -all configurations were S-shaped as predicted. Figure 4 shows the configuration for one of these posi-

\section{Figure 3}

Two-Dimensional Configuration of Unidimensional Distances Raised to the Power of .33

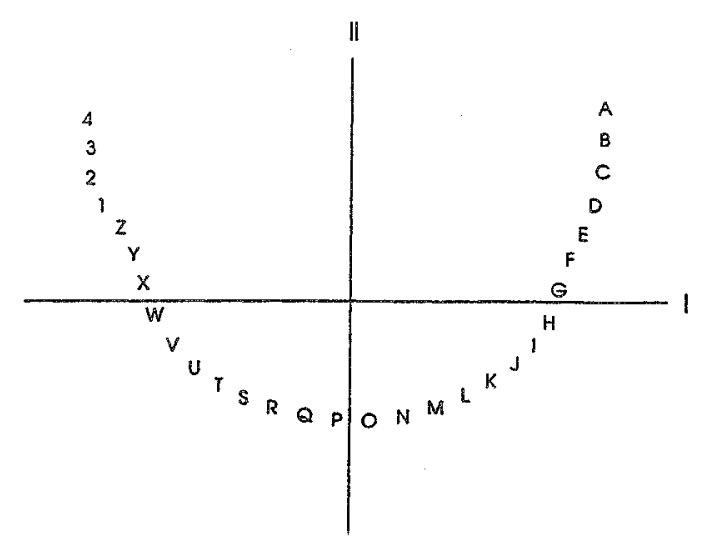

tively accelerating functions: the configuration for distances raised to the power 3.00 .

Figure 5 shows the configuration for the linear function. Rather than forming a line, as perhaps expected, the configuration is slightly $\mathrm{C}$-shaped.

These results suggest that when the stimulus set is unidimensional, the shape of the two-dimensional configuration is related to the form of the monotone function mapping distances onto dissimilarity data. Further, when the monotone function mapping distances onto the data is either positively or negatively accelerating, the configuration takes on a very distinctive $\mathrm{C}$ - or S-shape when the stimulus set is unidimensional.

The Shepard diagrams (Kruskal et al., 1973) for the one-dimensional solutions tend to confirm the conclusions that would be reached by looking at
Figure 4

Two-Dimensional Configuration of Unidimensional Distances Raised to the Power of 3.00

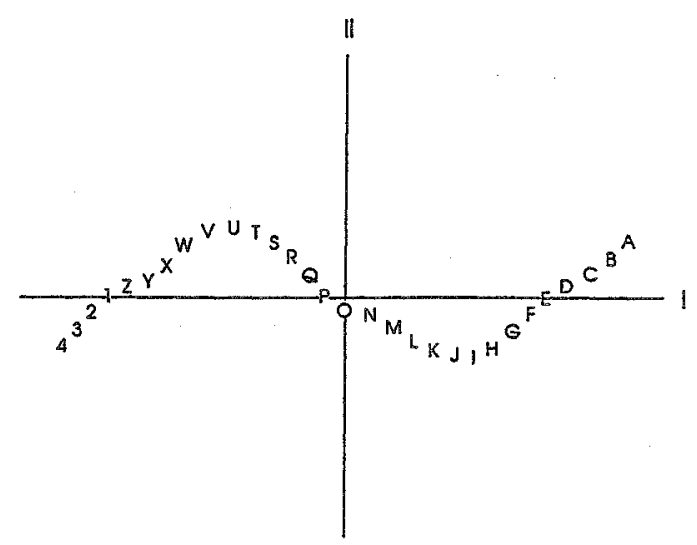

the two-dimensional configuration. A Shepard diagram is a scatter diagram that, for each proximity data point, shows the estimated distance (or the optimally rescaled data) plotted against the data.

Such a diagram shows the inverse of the transformation mapping distances onto the data. For powers of $.25, .33$, and .50 (negatively accelerating transformations), the one-dimensional Shepard diagrams (not shown) accurately portrayed the inverse of the transformation as a monotone increasing, positively accelerating function. Similarly, for powers of $2.0,3.0$, and 4.0 , the one-

\section{Figure 5}

Two-Dimensional Configuration of Unidimensional Distances

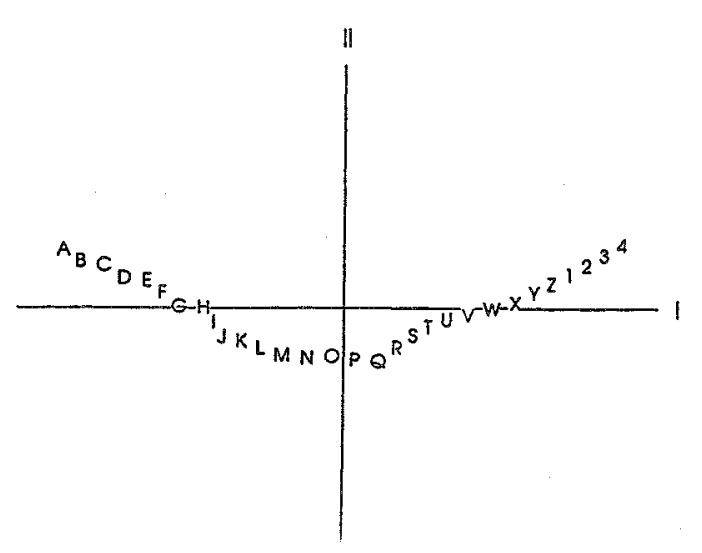


dimensional Shepard diagrams accurately portrayed the inverse of the transformation as a monotone increasing, negatively accelerating function. Thus, the shape of the two-dimensional configuration and the one-dimensional Shepard diagram represented two pieces of multidimensional scaling evidence that reflected the form of the monotone transformation mapping distances onto the data.

To examine the question of whether a similar pattern might occur in higher dimensionalities, a square grid of 49 points in two dimensions was created by crossing all possible integers from 1.0 to 7.0 for the first coordinate with all possible integers from 1.0 to 7.0 for the second coordinate. When distances for all possible pairs of the 49 points were computed and raised to a positive power, the resulting three-dimensional scaling (not shown) displayed no discernible pattern. However, when the distances were raised to fractional powers, the points formed a distinctive "bowl" shape.

This bowl shape can be seen in Figure 6, which shows Dimension 1 plotted against Dimension 2 and Dimension 1 plotted against Dimension 3. The basic grid can be seen in Figure 6a, and the curvature of the grid into a bowl shape in three dimensions can be seen in Figure 6b. The longest distances (that is, those between points at opposite corners of the grid) have been foreshortened by folding them up toward each other. Figure $6 \mathrm{~b}$ shows how the grid comers marked by asterisks in Figure 6a have been folded toward each other. In the plot of Dimension 2 against Dimension 3 (not shown), the corners marked by arrows are folded toward each other. It appears that for the two-dimensional C-shape of unidimensional stimulus sets and a negatively accelerating monotone transformation, there exists an analogous bowl shape in three dimensions for two-dimensional stimulus sets and a negatively accelerating monotone function.

Figure 7 displays the two-dimensional analysis of two datasets from Davison and Wood (1983). Davison and Wood showed that if paired-comparisons choice data satisfy either of the major unidimensional paired-comparisons models (Bradley \& Terry, 1952; Thurstone, 1927), then the proximity measure $\delta_{i j}=\left|\pi_{i j}-.50\right|$ will satisfy the as-
Fingure 6

Three-Dimensional Configuration of Two-Dimensional Distances

(a) Dimension 1 Against Dimension 2

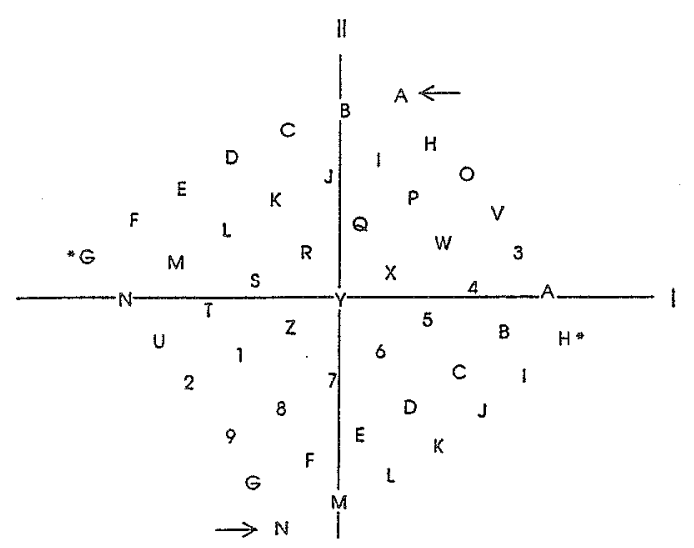

(b) Dimension 1 Against Dimension 3

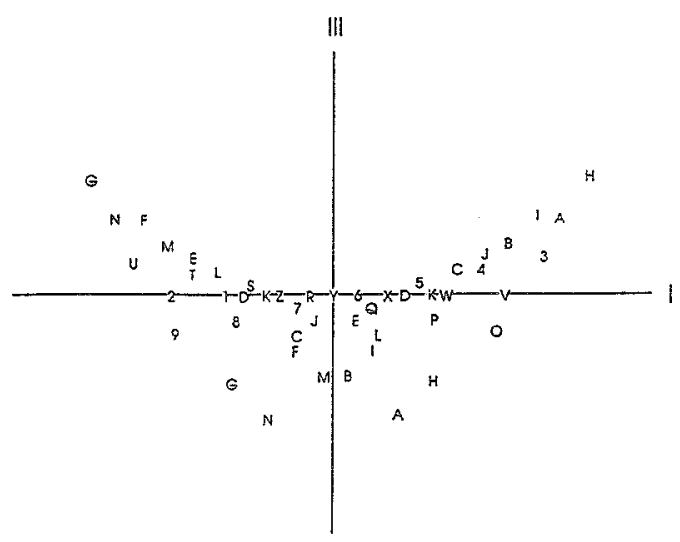

sumptions of a unidimensional nonmetric scaling model in the model scale values $x_{i}, x_{j}$. Here $\pi_{i j}$ is the proportion of persons selecting stimulus $j$ over stimulus $i$. Because both models posit an ogive function mapping stimulus differences $\left(x_{i}-x_{j}\right)$ onto choice probabilities $\pi_{i j}$, both models imply a negatively accelerating function mapping distances onto proximities. This supports the prediction that if the choice proportions satisfy either model, a two-dimensional scaling of proximities $\delta_{i j}=\left|\pi_{i j}-.50\right|$ will yield a $\mathrm{C}$-shape. 
Figure 7a shows results from an analysis of the classic Ayres handwriting data (Gulliksen \& Tukey, 1958). These do display the predicted C-shape, a result that confirms a hypothesis that these choice data satisfy a unidimensional choice model. Figure $7 \mathrm{~b}$ shows the analysis of Guilford's (1954) vegetable data. These points do not array themselves in the expected $\mathrm{C}$-shape, a result inconsistent with the hypothesis that they satisfy a unidimensional choice model.

As illustrated in the examples of Figure 7, examining a two-dimensional configuration of possibly unidimensional stimuli can be useful in two respects. First, it can be useful in deciding whether the stimuli are truly unidimensional, because when the transformation is positively or negatively accelerating, the stimuli tend to form very distinctive shapes when scaled in two dimensions. Second, when the stimuli are unidimensional, the shape of the two-dimensional configuration, along with the Shepard diagram, can be informative as to the nature of the monotone function relating distances to data.

\section{Discussion}

All analyses reported above were based on KYST's rational starting configuration, the TORSCA start. The choice of starting configuration appears to be important. When other starting configurations were used, the two-dimensional solutions formed $\mathrm{C}$ - or $\mathrm{S}$-shaped configurations, but the C-and S- shapes were not necessarily associated with positively and negatively accelerating functions in the predicted manner.

Why would the starting configuration matter? Roughly speaking, nonmetric scaling uses two features of the model to account for variance in the data: an estimated monotone transformation and stimulus coordinate estimates. When a rational starting configuration is used, the initial coordinates are estimated so as to account for as much variance in the data as possible, and the estimated monotone transformation is largely used to account for remaining variance. By folding the configuration of points as conjectured above, the starting
Figure 7

Two-Dimensional Configurations

(a) Based on the Ayres Handwriting Data

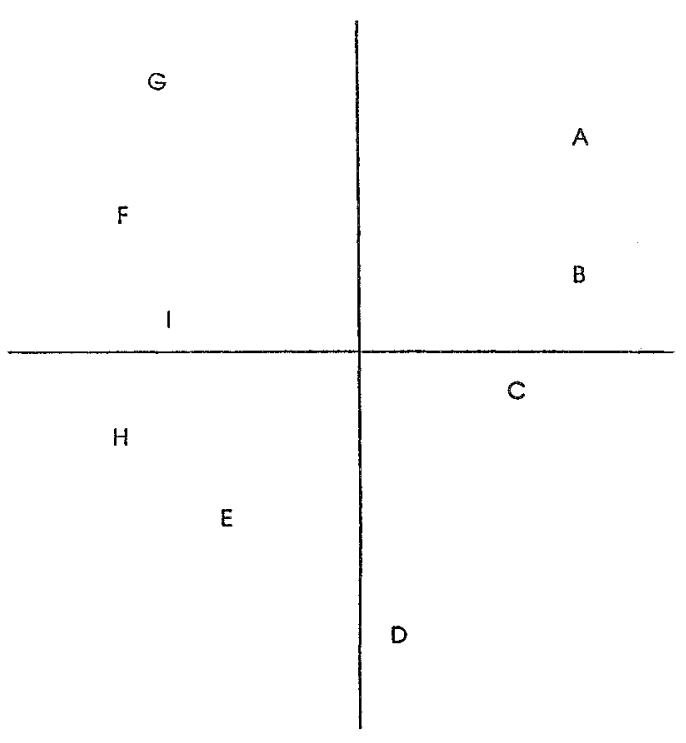

(b) Based on Guilford's (1954) Vegetable Data

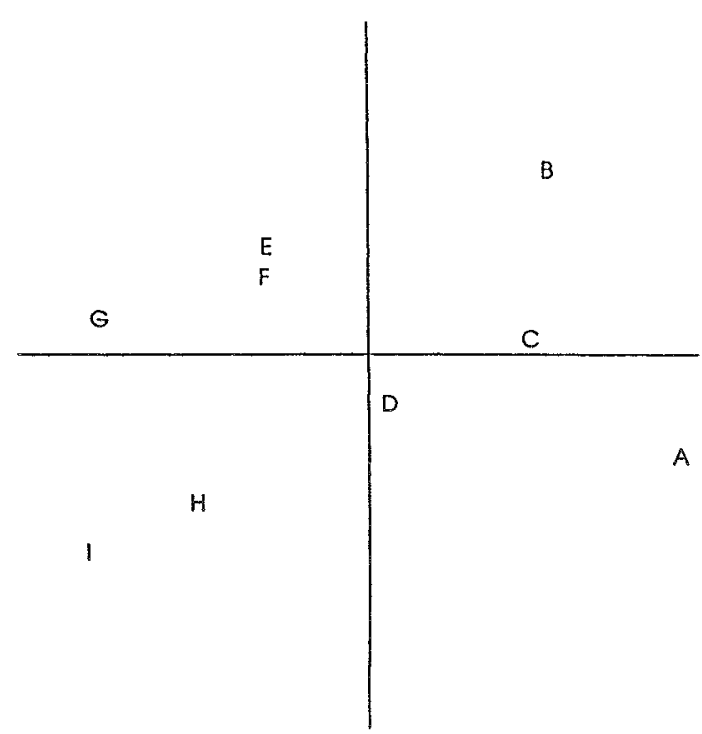

configuration will account for a maximum amount of variance in the data, and this folding gets carried over into the final configuration. When some other starting configuration is used, the estimated monotone transformation, not a folding of the stimulus 
configuration, may be used to account for the variance represented by the function mapping distances onto the data; hence, the folding need not fit the above predictions when other starting configurations are used.

In the multidimensional scaling model for psychological data, direct proximity measures are conceived as a mapping of psychological dissimilarity, represented as distances in the response model, onto a response scale. The response scale may take many forms, including ordered categories in a category rating task or response latencies in a same/ different judgment task. In some cases, the researcher will be interested in the nature of the function mapping psychological dissimilarity onto the response measure. When a rational staring configuration, such as the TORSCA start in KYST, is used and the stimulus sef is unidimensional, the twodimensional configuration can offer a clue as to the general form of the mapping.

Other authors (Davison, 1983; Heiser, 1981; Kruskal \& Wish, 1978; Shepard, 1974) have noted that the C- and S-shapes are an indication of unidimensionality. When the data are unidimensional and a rational starting configuration is used, the shape of the two-dimensional configuration also indicates the form of the function mapping distances onto data. The $\mathrm{C}$ - and S-shaped configurations are useful both in deciding dimensionality and in understanding how psychological similarities, as represented by model distances, are related to the data.

\section{References}

Bradley, R. A., \& Terry, M. E. (1952). Rank analysis of incomplete block designs: 1. The method of paired comparisons. Biometrika, 39, 324-345.

Davison, M. L. (1983). Multidimensional scaling. New York: Wiley.

Davison, M. L., \& Wood, P. K. (1983). Fitting unidimensional choice models with nonmetric multidimensional scaling. Applied Psychological Measurement, 7, 333-340.

Guilford, J. P. (1954). Psychometric methods. New York: McGraw-Hill.

Gulliksen, H., \& Tukey, J. (1958). Reliability for the law of comparative judgment. Psychometrika, 23, 95110.

Heiser, W. J. (1981). Unfolding analysis of proximity data. Leiden, The Netherlands: Reprodienst Psychologie.

Kruskal, J. B., \& Wish, M. (1978). Multidimensional scaling. Beverly Hills CA: Sage.

Kruskal, J. B., Young, F. W., \& Seery, J. B. (1973). How to use KYST, a very flexible program to do multidimensional scaling and unfolding (Unpublished manuscript). Murray Hill NJ: Bell Laboratories.

Shepard, R. N. (1974). Representation of structure in similarity data: Problems and prospects. Psychometrika, 39, 373-422.

Thurstone, $\mathbb{L}$. L. (1927). A law of comparative judgment. Psychological Review, 34, 273-286.

\section{Author's Address}

Send requests for reprints or further information to Mark L. Davison, Department of Educational Psychology, University of Minnesota, 178 Pillsbury Drive S.E., Minneapolis MN 55455, U.S.A. 